\title{
Reply to the Letter to the Editor of Yongzhao Zhao et al. concerning "Treating multi-level cervical disc disease with hybrid surgery compared to anterior cervical discectomy and fusion: a systematic review and meta-analysis" by Lu VM, Zhang L, Scherman DB et al. Eur Spine J (2016). doi:10.1007/s00586-015-4324-0
}

\author{
Victor M. Lu' ${ }^{1}$ Kevin Phan ${ }^{1,2}$ \\ Received: 9 October 2016/ Accepted: 13 October 2016/Published online: 19 October 2016 \\ (c) Springer-Verlag Berlin Heidelberg 2016
}

\section{Dear Editor,}

Thank you for the opportunity to respond to the Letter of the Editor regarding our recently published review "Treating multi-level cervical disc disease with hybrid surgery compared to anterior cervical discectomy and fusion: a systematic review and meta-analysis" published online in September 2016 in European Spine Journal [1].

The authors raise a valid argument for the use of random effects modelling. Unfortunately, this was not employed for this meta-analysis. With respect to the particular outcomes highlighted by the letter, it is worth comparing overall significance between meta-analyses. For operation time, the non-significant difference in Figure $1 \mathrm{~A}$ is in contrast to our review in Figure 2A. From a clinical perspective it is difficult to account for such variation in surgeon history, control and skillset and admittedly random effects would perhaps account for this better. For range of motion (ROM) in Figure 1B, both the overall trend and significance direction are consistent with our results in Figure 5A. For visual analogue scale (VAS) in Figure 1C, the authors did not stratify the outcomes based on 2- vs 3-level which introduces a level of difficulty in interpreting significance. This is in the context of a $p$ value of 0.03 statistically, as well as an absolute value difference in VAS of one unit clinically. Accounting for the heterogeneity by subgroup analysis as in our Figure 5B, the trend proposed does not hold for 2-level operations.

Victor M. Lu

vilu6071@uni.sydney.edu.au

1 Sydney Medical School, The University of Sydney, Sydney, NSW 2006, Australia

2 NeuroSpine Surgery Research Group (NSURG), Prince of Wales Private Hospital, Sydney, Australia
It is also worth mentioning that controlling directly for the heterogeneity that was 2- vs 3-level operations was not straight forward. As acknowledged in our review, there was one study in which 2- vs 3-level operations were not completely discernible. We felt that although this would compromise the heterogeneity of some results as highlighted by this letter, it would be outweighed by the benefit of being able to propose more conclusions for future investigation, again as highlighted by this letter.

The use of hybrid surgery to treat multi-level cervical disc disease is still a developing field of interest. As a result, the quality of published studies requires review. This was performed in our review according to the MOOSE Criteria as shown in Table 3 which assessed for bias risk. As the authors of the letter correctly highlight, currently the cohort for study remains small. To exclude studies with small bias, risk will compromise the significance of conclusions for future studies which requires careful consideration.

We are in total agreement with the authors that there is a strong need for an appropriately designed randomised controlled trial. This indeed was our conclusion, and interpretation of our results should be done with caution until this occurs.

Compliance with ethical standards

Conflict of interest None.

\section{Reference}

1. Lu VM, Zhang L, Scherman DB et al (2016) Treating multi-level cervical disc disease with hybrid surgery compared to anterior cervical discectomy and fusion: a systematic review and metaanalysis. Eur Spine J (2016). doi:10.1007/s00586-015-4324-0 